\title{
Niveles de satisfacción estudiantil en una facultad de ingeniería por la virtualización de la enseñanza durante la pandemia de COVID 19
}

Max Clive Alcántara Trujillo

malcantara@uncp.edu.pe

Eli Teobaldo Caro Meza

ecaro@uncp.edu.pe

Mensia Basilia Solis Llallico

msolis@uncp.edu.pe

Helmer Lopez Gutierrez

hlopez@uncp.edu.pe

Universidad Nacional del Centro del Perú

Huancayo - Perú

\section{RESUMEN}

Objetivo: Determinar los niveles de satisfacción estudiantil en una Facultad de Ingeniería por la virtualización de la enseñanza durante la pandemia de COVID-19.

Sujetos y métodos: Estudio observacional, cuantitativo, prospectivo y transversal. Se aplicó un cuestionario virtual, basado en la escala tipo Likert, validado por juicio de expertos con una fiabilidad alfa de Cronbach de 0,865 . Con un muestreo probabilístico aleatorio estratificado de noviembre 2020 a febrero de 2021.

Conclusión. El nivel de satisfacción preponderante en las subdimensiones estudio individual el 57,6\% algunas veces los estudiantes han tenido dificultades en sus estudios, trabajo en grupo $64,3 \%$ ha percibido tener dificultades, estar en clases virtuales representa el 75,6\%, dar opinión en clases $62,7 \%$, rendir evaluaciones 70,4\%, entregar tareas 59,2\%, comunicación con los docentes de la FIMM 64,3\%, comunicación con compañeros de clases $53,1 \%$. En el estado de ánimo que predomina en la subdimensión los estudiantes presentan aburrimiento medio 46,4\%, irritabilidad medio 42,8\%, miedo 43,8\%, negatividad 40,3\%, paciencia 47,9\%, serenidad medio 50,0\%, tristeza medio 42,3\%.; en la distribución de su tiempo el 49,5\% de los estudiantes se dedican a labores domésticas, en los estudios virtuales el $49 \%$ se encuentra estresado, el equipo de ingreso a la plataforma virtual es con celular 65,3\%, problemas de conexión con la plataforma virtual de la universidad $55,1 \%$ por variaciones de conectividad, desempeño laboral docente 62 , $2 \%$ regular.

Palabras clave: COVID-19; Enseñanza; Estudiantes de ingeniería; Pandemia; Satisfacción; Virtualización. 


\title{
Student satisfaction levels in an engineering college due to the virtualization of teaching during the COVID 19 pandemic
}

\begin{abstract}
Objective: To determine the levels of student satisfaction in a Faculty of Engineering for the virtualization of teaching during the COVID-19 pandemic.

Subjects and methods: Observational, bquantitative, prospective and cross-sectional study. A virtual questionnaire was applied, based on the Likert-type scale, validated by expert judgment with a Cronbach's alpha reliability of 0.865 . With a stratified random probability sampling from November 2020 to February 2021.

Conclution: The prevailing level of satisfaction in the individual study subdimensions $57.6 \%$. Sometimes students have had difficulties in their studies, group work $64.3 \%$ have perceived difficulties, being in virtual classes represents $75.6 \%$, giving opinion in classes $62.7 \%$, take evaluations $70.4 \%$, deliver homework 59.2\%, communication with FIMM teachers $64.3 \%$, communication with classmates $53.1 \%$. In the mood that predominates in the subdimension, students present average boredom $46.4 \%$, average irritability $42.8 \%$, fear $43.8 \%$, negativity $40.3 \%$, patience $47.9 \%$, average serenity $50.0 \%$, average sadness $42.3 \%$; In the distribution of their time, $49.5 \%$ of the students dedicate themselves to housework, in virtual studies $49 \%$ are stressed, the team entering the virtual platform is with a cell phone $65.3 \%$, connection problems $55.1 \%$ with the university's virtual platform due to variations in connectivity, 62 teacher job performance, $2 \%$ regular.
\end{abstract}

Keywords: COVID-19; Teaching; Engineering students; Pandemic; Satisfaction; Virtualization

Artículo recibido: 05 de Mayo 2021 Aceptado para publicación: 20 de Junio 2021 Correspondencia: malcantara@uncp.edu.pe Conflictos de Interés: Ninguna que declarar 


\section{INTRODUCCIÓN}

La Organización Mundial de la Salud informó del brote de la enfermedad por el nuevo coronavirus (COVID-19) notifi cado por primera vez en la ciudad de Wuhan en China el 31 de diciembre de 2019. El aumento de casos fuera de China se multiplicó por 13 y el número de países afectados en poco tiempo se triplicó. Preocupada por los niveles alarmantes de propagación y gravedad, el 11 de marzo la Organización Mundial de la Salud (OMS,2020) declaró la pandemia. Frente a esta comunicación, el Gobierno peruano proclamó la emergencia sanitaria y decretó medidas de prevención y control, por lo que el Ministerio de Educación dictaminó que las entidades educativas públicas y privadas de educación superior postergaran o suspendieran sus actividades académicas y administrativas (Diario el Peruano, 2020). En esos momentos, en la Facultad de Ingeniería Metalúrgica y de Materiales (FIMM) de la Universidad Nacional del Centro del Perú (UNCP), varios estudiantes venían desarrollando actividades académicas, por lo que la institución tomó la decisión de continuarlas, con la implementación de una plataforma virtual para el desarrollo del proceso de enseñanza-aprendizaje, así como con la capacitación de los docentes y estudiantes en el uso de este nuevo entorno virtual. Al ser súbita esta transición novedosa, se logró evidenciar algunas dificultades propias de esta nueva tecnología, pero también muchas ventajas significativas. Algunos estudios concluyen que, debido a los avances tecnológicos, los docentes tienen una variedad de herramientas para mejorar y optimizar su práctica docente con las exigencias que los estudiantes de hoy en día demandan (Nereyda, et al., 2018). Existen diferentes grados de satisfacción de los estudiantes respecto a cómo se sienten con la incorporación del aula virtual a sus asignaturas, y se ha observado que incluso algunos mejoran su rendimiento académico, mientras que los que no poseen ordenador o acceso a internet por problemas económicos ven complicado su aprendizaje (Suasti C, 2020). La satisfacción de los estudiantes respecto al método, los contenidos y, principalmente, la comunicación en línea con el docente es parte del éxito de los programas virtuales. La calidad educativa del método virtual trasciende no sólo a la experiencia de transmitir y compartir la información entre docentes y estudiantes, sino también a la satisfacción de los usuarios ( Boullosa, et al., 2017). Existen múltiples evidencias de que la implementación del entorno virtual en la educación universitaria en ingeniería metalúrgica ha generado considerables resultados exitosos en los estudiantes con altos grados de satisfacción (Díaz, et al 2015). 
Por lo referido y el cambio súbito del proceso de enseñanza-aprendizaje de presencial a virtual en el contexto de la pandemia por la COVID-19, fue oportuno determinar los niveles de satisfacción con esta nueva estrategia educativa en los estudiantes de la Facultad de Ingeniería Metalúrgica y de materiales de la UNCP durante el presente semestre académico.

\section{ESTRATEGIAS METODOLÓGICAS O MATERIALES Y MÉTODOS}

Antes de la ejecución del presente estudio, se obtuvo el permiso institucional por parte del decano de la facultad y el dictamen favorable del comité de ética. El estudio fue observacional, cuantitativo, prospectivo y transversal, y consistió en la aplicación de un cuestionario virtual mediante el formulario de Google Forms a los estudiantes de la Facultad de Ingeniería Metalúrgica y de Materiales de la UNCP entre noviembre 2020 y febrero 2021, para medir su satisfacción con la educación virtual (Valdez E. 2018). El cuestionario está basado en una escala tipo Likert en la dimensión nivel de satisfacción: No he tenido dificultades, algunas veces he tenido dificultades, siempre tengo dificultades. En estado de ánimo muy bajo, bajo, medio, alto, muy alto. En la subdimensión distribución de tiempo: labores domésticas, estudio en casa, actividades de ocio, trabajar para ayudar en casa. Estudio virtual: confundido, contento, estresado, aburrido, ninguno. Equipo de ingreso a la plataforma virtual de la universidad: ordenador, laptop, Tablet, celular. Problemas de conexión con la plataforma virtual de la universidad: acceso a internet en general, acceso a las clases en línea, problemas con micrófono, problemas con la cámara, variaciones de conectividad. Desempeño laboral docente: bueno, regular, malo. El cuestionario fue sometido a juicio por expertos para la validación con resultado de muy alta validez. En el análisis estadístico de fiabilidad, resultó con un alfa de Cronbach de 0,865. La población de estudio estuvo constituida por los 350 estudiantes matriculados en el semestre 2020-I y 20020-II, y después del muestreo probabilístico aleatorio estratificado del $3 .^{\circ}$ al $10{ }^{\circ}$ ciclo académico se obtuvo una muestra de 196 estudiantes que aceptaron voluntariamente, mediante el consentimiento informado, ser parte del presente estudio. Finalizada la recolección de los datos, se procedió a ordenarlos en las dimensiones de cada variable en concordancia con los objetivos del presente estudio; asimismo, se generó una base de datos y se procesó con el programa estadístico SPSS v.25. Se realizó el análisis estadístico descriptivo de las variables, y se expresaron en frecuencia, porcentaje, media y desviación estándar. 


\section{RESULTADOS Y DISCUSIÓN}

La muestra estuvo constituida por 196 estudiantes (15,30\% de sexo femenino), con una edad media de 22,6 \pm 5 años, que aceptaron ser parte de la muestra mediante consentimiento informado. En la dimensión nivel de satisfacción, en su subdimensión estudio individual el (57.6\%) de los estudiantes ha tenido dificultades en realizar sus estudios, $35,7 \%$ no ha tenido dificultades, $6,7 \%$ tiene dificultades; en trabajo en grupo: $64,3 \%$ ha tenido dificultades, no ha tenido dificultades $26,0 \%$, siempre tiene dificultades 9,7\%; estar en clases virtuales, $75,6 \%$ tuvo dificultades, el 17,3\% no ha tenido dificultades y el 7,1\% siempre tiene dificultades; dar mi opinión en clases, el 62,7 de los estudiantes encuestados algunas veces ha tenido dificultades, el 30,1\% no ha tenido dificultades, $7,2 \%$ tiene dificultades; en rendir evaluaciones $70,4 \%$ ha tenido dificultades, el $20,4 \%$ no ha tenido dificultades, el 9,2\% tiene dificultades; entregar las tareas, el 59,2\% ha tenido dificultades, el 6,6\% no ha tenido dificultades; en la comunicación con los docentes, el $64,3 \%$ algunas veces ha tenido dificultades, el $28,5 \%$ no ha tenido dificultades, el 7,2\% siempre tiene dificultades; comunicación con compañeros de clases, el 53,1\% algunas veces ha tenido dificultades, el $42,3 \%$ no ha tenido dificultades, $4,6 \%$ siempre tiene dificultades. En la dimensión estado de ánimo, en sus subdimensiones aburrimiento fue: muy bajo $10,7 \%$, bajo $18,8 \%$, medio $46,4 \%$, alto $20,4 \%$; irritabilidad muy bajo $8,6 \%$, bajo $27,5 \%$, medio $42,8 \%$, alto $18,3 \%$, muy alto $2,8 \%$; Miedo muy bajo $10,2 \%$, bajo $35,2 \%$, medio $43,8 \%$, alto $8,6 \%$, muy alto $2,2 \%$; negatividad muy bajo $13,7 \%$, bajo $32,1 \%$, medio $40,3 \%$, alto $10,7 \%$, muy alto $3,2 \%$; paciencia muy bajo $8,6 \%$, bajo $29,5 \%$, medio $47,9 \%$, alto $10,2 \%$, muy alto $3,8 \%$; serenidad muy alto $7,6 \%$, bajo $28,1 \%$, medio $50,0 \%$, alto $10,2 \%$, muy alto $4,1 \%$; tristeza muy alto $12,7 \%$, bajo $31,1 \%$, medio $42,3 \%$, alto $9,7 \%$, muy alto 4,2\%. En la dimensión distribución de tiempo: el 43,9\% se dedica a labores domésticas, el 31,!\% estudiar en casa, 6,6\% actividades de ocio, 18,4\% a trabajar para ayudar en casa. En la dimensión familiaridad e impacto en las herramientas virtuales, en sus subdimensiones fue; estudio virtual confundido $13,3 \%$, contento $22,4 \%$, estresado $49,5 \%$, aburrido $11,2 \%$, ninguno $3,6 \%$; equipo de ingreso a la plataforma virtual de la Universidad con ordenador $7,1 \%$, laptop $27,0 \%$, tablet $0,6 \%$, celular $65,3 \%$, problemas de conexión con la plataforma virtual de la universidad acceso a internet en general 22 , $4 \%$, acceso a las clases en línea 12,2\%, problemas con el micrófono 7,2\%, problemas con 
la cámara 3,1\%, variaciones de conectividad 55,1\%; desempeño laboral docente bueno $31,1 \%$, regular $62,2 \%$, malo $6,7 \%$.

La muestra estuvo constituida por 196 estudiantes: las características de sexo y edad se detallan en la tabla I; la distribución de los estudiantes por ciclo académico, en la tabla II; y el nivel de satisfacción por dimensiones, en la tabla III.

Tabla I: Distribución de los estudiantes por sexo y edad

\begin{tabular}{cllll}
\hline \multirow{2}{*}{ Sexo } & & $\mathbf{n}$ & $\mathbf{\%}$ \\
\cline { 2 - 4 } & Femenino & 30 & 15,3 \\
\cline { 2 - 4 } Rango de edad & Masculino & 166 & 84,7 \\
\hline \multirow{3}{*}{ 19-22 } & 131 & 66,8 \\
& $23-26$ & 37 & 18,9 \\
\cline { 2 - 4 } & $15-18$ & 22 & 11,2 \\
\hline
\end{tabular}

Fuente: Elaboración propia

Tabla II: Distribución de los estudiantes por semestre académico $(n=196)$

\begin{tabular}{ccc} 
Semestre & n & \% \\
\hline $3^{\circ}$ & 29 & 14,8 \\
$4^{\circ}$ & 54 & 27,6 \\
$5^{\circ}$ & 30 & 15,3 \\
$6^{\circ}$ & 23 & 11,7 \\
$7^{\circ}$ & 34 & 17,3 \\
$8^{\circ}$ & 9 & 4,6 \\
$9^{\circ}$ & 16 & 8,2 \\
$10^{\circ}$ & 1 & 0,5 \\
\hline
\end{tabular}

Fuente: Elaboración propia

Tabla III: Dimensión nivel de satisfacción

\begin{tabular}{llcc}
\hline Subdimensión & \multicolumn{1}{c}{ Factores } & n & \% \\
\hline \multirow{3}{*}{ Estudio individual } & No he tenido dificultades & 70 & 35,7 \\
& Algunas veces he tenido dificultades & 113 & 57,6 \\
& Siempre tengo dificultades & 13 & 6,7 \\
\hline \multirow{3}{*}{ Trabajo en grupo } & No he tenido dificultades & 51 & 26,0 \\
& Algunas veces he tenido dificultades & 126 & 64,3 \\
& Siempre tengo dificultades & 19 & 9,7 \\
\hline \multirow{2}{*}{ Estar en clases } & No he tenido dificultades & 34 & 17,3 \\
virtuales & Algunas veces he tenido dificultades & 150 & 75,6 \\
& Siempre tengo dificultades & 12 & 7,1 \\
\hline
\end{tabular}




\begin{tabular}{|c|c|c|c|}
\hline \multirow{3}{*}{$\begin{array}{l}\text { Dar mi opinión en } \\
\text { clases }\end{array}$} & No he tenido dificultades & 59 & 30,1 \\
\hline & Algunas veces he tenido dificultades & 123 & 62,7 \\
\hline & Siempre tengo dificultades & 14 & 7,2 \\
\hline \multirow{3}{*}{ Rendir evaluaciones } & No he tenido dificultades & 40 & 20,4 \\
\hline & Algunas veces he tenido dificultades & 138 & 70,4 \\
\hline & Siempre tengo dificultades & 18 & 9,2 \\
\hline \multirow{3}{*}{ Entregar tareas } & No he tenido dificultades & 67 & 34,2 \\
\hline & Algunas veces he tenido dificultades & 116 & 59,2 \\
\hline & Siempre tengo dificultades & 13 & 6,6 \\
\hline \multirow{3}{*}{$\begin{array}{l}\text { Comunicación con } \\
\text { los docentes de la } \\
\text { FIMM }\end{array}$} & No he tenido dificultades & 56 & 28,5 \\
\hline & Algunas veces he tenido dificultades & 126 & 64,3 \\
\hline & Siempre tengo dificultades & 14 & 7,2 \\
\hline \multirow{3}{*}{$\begin{array}{l}\text { Comunicación con } \\
\text { compañeros de } \\
\text { clases }\end{array}$} & No he tenido dificultades & 83 & 42,3 \\
\hline & Algunas veces he tenido dificultades & 104 & 53,1 \\
\hline & Siempre tengo dificultades & 9 & 4,6 \\
\hline
\end{tabular}

Fuente: Elaboración propia

Tabla IV: Dimensión estado de ánimo de los estudiantes de la FIMM-UNCP

\begin{tabular}{llccc}
\hline Subdimensión & Factores & n & \% \\
\hline \multirow{5}{*}{ Aburrimiento } & Muy bajo & 21 & 10,7 \\
& Bajo & 37 & 18,8 \\
& Medio & 91 & 46,4 \\
& Alto & 40 & 20,4 \\
& Muy alto & 7 & 3,7 \\
\hline \multirow{5}{*}{ Irritabilidad } & Muy bajo & 17 & 8,6 \\
& Bajo & 54 & 27,5 \\
& Medio & 84 & 42,8 \\
& Alto & 36 & 18,3 \\
& Muy alto & 5 & 2,8 \\
\hline \multirow{5}{*}{ Miedo } & Muy bajo & 20 & 10,2 \\
& Bajo & 69 & 35,2 \\
& Medio & 86 & 43,8 \\
& Alto & 17 & 8,6 \\
& Muy alto & 4 & 2,2 \\
\hline & Muy bajo & 27 & 13,7 \\
& Bajo & 63 & 32,1 \\
& Medio & 79 & 40,3 \\
& Alto & 21 & 10,7 \\
& Muy alto & 6 & 3,2 \\
\hline
\end{tabular}




\begin{tabular}{llcc}
\hline & Muy bajo & 17 & 8,6 \\
Paciencia & Bajo & 58 & 29,5 \\
& Medio & 94 & 47,9 \\
& Alto & 20 & 10,2 \\
& Muy alto & 7 & 3,8 \\
\hline \multirow{5}{*}{ Serenidad } & Muy bajo & 15 & 7,6 \\
& Bajo & 55 & 28,1 \\
& Medio & 98 & 50,0 \\
& Alto & 20 & 10,2 \\
Tristeza & Muy alto & 8 & 4,1 \\
\hline & Muy bajo & 25 & 12,7 \\
& Bajo & 61 & 31,1 \\
& Medio & 83 & 42,3 \\
& Alto & 19 & 9,7 \\
& Muy alto & 8 & 4,2 \\
\hline
\end{tabular}

Fuente: Elaboración propia

Tabla V: Dimensión familiaridad e impacto de herramientas virtuales $(n=196)$

\begin{tabular}{llcc}
\hline \multicolumn{1}{c}{ Dimensión } & \multicolumn{1}{c}{ Factores } & n & \% \\
\hline \multirow{4}{*}{$\begin{array}{l}\text { Distribución de } \\
\text { tiempo }\end{array}$} & Labores domésticas & 86 & 43,9 \\
& Estudio en casa & 60 & 31,1 \\
& Actividades de ocio & 13 & 6,6 \\
& Trabajar para ayudar en casa & 37 & 18,4 \\
\hline \multirow{2}{*}{ Estudio virtual } & Confundido & 26 & 13,3 \\
& Contento & 45 & 22,4 \\
& Estresado & 96 & 49,5 \\
Equipo de ingreso a la & Aburrido & 22 & 11,2 \\
plataforma virtual de & Ninguno & 7 & 3,6 \\
\hline la FIMM-UNCP & Taptop & 14 & 7,1 \\
& Celular & 53 & 27,0 \\
Problemas de & Acceso a internet en general & 2 & 0,6 \\
conexión con la & Acceso a las clases en línea & 127 & 65,3 \\
\hline plataforma virtual de & Problemas con el micrófono & 45 & 22,4 \\
la FIMM-UNCP & Problemas con la cámara & 24 & 12,2 \\
& Variaciones de conectividad & 14 & 7,2 \\
\hline \multirow{2}{*}{$\begin{array}{l}\text { Desempeño laboral } \\
\text { docente }\end{array}$} & Bueno & 6 & 3,1 \\
& Regular & 107 & 55,1 \\
\hline
\end{tabular}

Fuente: Elaboración propia 


\section{DISCUSIÓN}

Los resultados del estudio demostraron que los niveles de satisfacción de los estudiantes ante la virtualización del proceso de enseñanza-aprendizaje alcanzó un nivel medio en los ocho semestres académicos en los cuales se investigaron las variables de estudio, que fueron la percepción de la educación virtual y el nivel de satisfacción resultante del proceso de virtualización de la enseñanza. La virtualización del proceso de enseñanzaaprendizaje posee un carácter complejo y dinámico, además de altamente exigente, sobre todo en instituciones donde se implementa por primera vez, y resulta, por tanto, innovadora y muy dependiente de la capacidad, por un lado, de la institución para poner a disposición herramientas virtuales pertinentes e idóneas, y, por otro, de contar con docentes capaces de asumir el reto de la virtualización del proceso, situación que ya había sido advertida en anteriores investigaciones (Díaz, et al 2015). Los resultados obtenidos muestran que la familiaridad e impacto de las herramientas virtuales fue la dimensión con mayores problemas de conexión. Ello reafirma el importante papel que desempeña el docente en el proceso de virtualización de la enseñanza. Por tanto, es necesario invertir de manera decidida en la capacitación y, con ello, en la adquisición de mayores y mejores competencias en el empleo de herramientas virtuales por parte de los docentes, acompañado de la capacitación en la implementación de estrategias de enseñanza virtual que permitan a los estudiantes la adquisición de saberes nuevos que ellos consideren de un nivel adecuado y alto. La satisfacción del estudiante es el elemento básico y fundamental para lograr institucionalmente una formación superior con el objetivo de alcanzar y superar las expectativas del estudiante que hace uso de sus servicios. Por ello, es de gran importancia disponer de estos dos elementos claves: adecuadas herramientas virtuales y docentes altamente capacitados para tener un desempeño idóneo en el ámbito virtual; así, en el presente estudio se muestra un regular porcentaje de desempeño laboral docente, lo que refleja el papel que éste cumple en los procesos de enseñanza virtual, situación que ya había sido relacionada (Gros B. 2011). Del mismo modo, se debe entender que la satisfacción de los estudiantes es un factor fundamental en el grado de motivación, compromiso y niveles de aprendizaje que alcanzan, ya que la generación de un círculo virtuoso de satisfacción y motivación será de especial importancia para el logro de competencias por su parte, algo que ya otras investigaciones concluyeron previamente (Zambrano J. 2016). Este estudio tiene fortalezas y limitaciones. En cuanto a las primeras, 
se empleó un instrumento de medición válido y fiable; del mismo modo, al haberse realizado un muestreo probabilístico aleatorio estratificado, brinda una auténtica idea de la percepción que poseen los estudiantes de los diferentes ciclos académicos de formación ingenieril y los resultados se pueden extrapolar a la población de estudiantes de la Facultad de Ingeniería Metalúrgica y de Materiales de la UNCP. Entre sus limitaciones se podría mencionar el hecho de que sólo presenta información desde el punto de vista estudiantil, y por ello se hace recomendable explorar este tema desde otros ángulos, como el docente o el institucional, lo que permitiría obtener una visión global del proceso de virtualización de la enseñanza-aprendizaje, sobre todo en un área tan crítica y trascendente como la formación ingenieril en el contexto de una pandemia. El adecuado análisis de los resultados obtenidos en cuanto a la educación virtual en sus dimensiones, empleo de recursos para el aprendizaje, acompañamiento y colaboración, todo ello con un carácter virtual, muestra que deben potenciarse, en razón de la percepción que poseen los estudiantes de contar con docentes motivados y con un alto nivel de compromiso para la implementación del proceso de enseñanza-aprendizaje virtual. Finalmente, es necesario investigar en qué medida, a largo plazo, el empleo cada vez mayor de herramientas y estrategias de carácter virtual impactará en el logro de competencias en los estudiantes de ingeniería metalúrgica y de materiales del nivel de pregrado.

\section{CONCLUSIÓN O CONSIDERACIONES FINALES}

El nivel de satisfacción preponerante en las subdimensiones estudio individual el 57,6\% algunas veces los estudiantes han tenido dificultades en sus estudios, trabajo en grupo 64,3\% ha percibido tener dificultades, estar en clases virtuales representa el 75,6\%, dar opinión en clases 62,7\%, rendir evaluaciones 70,4\%, entregar tareas 59,2\%, comunicación con los docentes de la FIMM 64,3\%, comunicación con compañeros de clases $53,1 \%$. En el estado de ánimo que predomina en la subdimensión los estudiantes presentan aburrimiento medio 46,4\%, irritabilidad medio 42,8\%, miedo 43,8\%, negatividad 40,3\%, paciencia 47,9\%, serenidad medio 50,0\%, tristeza medio 42,3\%.; en la distribución de su tiempo el 49,5\% de los estudiantes se dedican a labores domésticas, en los estudios virtuales el $49 \%$ se encuentra estresado, el equipo de ingreso a la plataforma virtual es con celular 65,3\%, problemas de conexión con la plataforma virtual de la universidad $55,1 \%$ por variaciones de conectividad, desempeño laboral docente 62 , $2 \%$ regular. 


\section{LISTA DE REFERENCIAS}

World Health Organisation. WHO (2020) Director-General's opening remarks at the World Health Assembly. Recuperado de: https://www. who.int/dg/speeches/detail/who-director-general-s-opening- remarks-at-theworld-health-assembly.

Decreto Supremo que declara en Emergencia Sanitaria a nivel nacional por el plazo de noventa (90) días calendario y dicta medidas de prevención y control del COVID19 (2020). Diario Oficial El Peruano. Recuperado de: https://busquedas.elperuano.pe/ normaslegales/decreto-supremo-que-declara-enemergencia- sanitaria-a-nivel-decreto-supremo-n-008-2020-sa-1863981-2/.

Nereyda S, Zazueta C, Soto M, Jiménez C. (2018). Satisfacción en el uso del aula virtual en estudiantes de medicina. Rev Deb en Eva y Curr ; 4: 2462-72.

Suasti C. (2020). Satisfacción de los estudiantes de la enseñanza superior con las clases virtuales. Ecuador: Instituto Politécnico de Leiria/Universidad Técnica de Manabí;

2018.NRecuperadoen:nhttps://iconline.ipleiria.pt/bitstream/10400.8/3641/1/UPT IC_Relatorio-CLAUDIA\%20SUASTI_27agosto18.pdf.

Boullosa C, Bustamante P, Juzcamaita H. (2020). Satisfacción del uso del aula virtual en estudiantes de segunda especialización del instituto de educación superior tecnológico público del ejército. Santiago de Surco, Perú: Universidad Marcelino Champagnat; 2017. Recuperado en: http://repositorio.umch.edu.pe/ handle/UMCH/429.

Paz C.(2018). La enseñanza de la patología a través del diseño de casos clínicopatológicos en estudiantes de medicina de una universidad compleja. Concepción, Chile: Universidad de Concepción.

Cuadrao L.(2016). El método de enseñanza virtual y su influencia en el aprendizaje de histopatología. Lima: Universidad Nacional Mayor de San Marcos.

Valdez E.(2018). La educación virtual y la satisfacción del estudiante en los cursos virtuales del instituto nacional materno perinatal. Trujillo, Perú: Universidad César Vallejo.

Fernández R, De la Mata-García M, Torres-Gómez A, RománGómez J, Pérez-Martínez P, Ruiz-Moral R, et al. (2018). La enseñanza virtual de imágenes clínicas, 
tutorizada mediante correo electrónico, es más eficiente que la enseñanza tradicional. Educ Med.; 11: pp.29-35.

Asturizaga-Rodríguez D. (2018). Satisfacción de los estudiantes con las nuevas tecnologías de la información y la comunicación en la cátedra de ginecología. Cuadernos Hospital de Clínicas ; 59: pp. 60-7.

Castillo T, Espinosa A, Soto C, Bustamante P. (2006). Evaluación de satisfacción del módulo de autoenseñanza para el estudio del sedimento urinario en una plataforma tecnológica. Educ Med ; 9: pp. 212-20.

Díaz K, Miguel, Landaeta I, Ortiz M, Fernández M. (2015). Satisfacción estudiantil con el uso de estrategias instruccionales apoyadas en TIC para el aprendizaje de bioquímica. Docencia Universitaria 2014; 15: 3-16. 13.

Gros B. (2011). Evolución y retos de la educación virtual. Construyendo el e-learning del siglo XXI. Barcelona: Editorial UOC.

Zambrano J. (2016). Factores predictores de la satisfacción de estudiantes de cursos virtuales. RIED, Revista Iberoamericana de Educación a Distancia; 19: pp. 21735.

15. Miguel R. Mercado-Rey, Santiago A. Cortez-Orellana, Richard J. Febres-Ramos (2020). Satisfacción estudiantil en una facultad de medicina por la virtualización de la enseñanza en el contexto de la pandemia de COVID-19. UPLA . 